\title{
The study of $\delta$ Scuti stars in the transition era from ground-based to space photometry
}

\author{
Rafael Garrido \\ Instituto de Astrofísica de Andalucía-CSIC, Apartado 3004, 18080 \\ Granada, Spain \\ Ennio Poretti \\ INAF-Osservatorio Astronomico di Brera, Via E. Bianchi 46, 23807 \\ Merate, Italy
}

\section{Introduction}

The different research teams involved in the study of $\delta$ Sct stars have slightly changed their strategy in the past years. The observational effort to secure worldwide coverage of case studies has been continued, but the requirements have become more severe, especially about target characterization and frequency resolution.

After the successful launch of the Canadian satellite MOST, which will be the pioneer of asteroseismology from space, the future missions are programmed to properly take into account the need for adequate frequency resolution: COROT will spent 30 and $150 \mathrm{~d}$ (additional and core programs, respectively) on the target, while EDDINGTON will spend up to $3 \mathrm{yr}$. Such a requirement is a direct consequence of the observational results on $\delta$ Sct, $\gamma$ Dor, SPB, and other stars obtained from ground. It should be noted that without these results (see Poretti 2000 for a review about $\delta$ Sct stars) the scientific background of the space missions would be much less defined and the risks of incomplete results (owing to inaccurate selection of targets, insufficient resolution, underestimate of the influence of the rotation) much higher.

\section{The increasing number of frequencies detected in FG Vir}

López de Coca et al. (1984) easily detected the main period of FG Vir on the basis of few nights. It can be considered the discovery step. After that, Mantegazza, Poretti \& Bossi (1994) detected seven frequencies on the basis of a 14-night run at the European Southern Observatory. It can be considered the second step, i.e., the limit for a single-site campaign. Owing to its equatorial position, FG Vir was a suitable candidate for a multisite campaign, since it is possible to observe it from both hemispheres. Indeed, Breger et al. (1999) confirmed the seven frequencies and increased the total number of detected frequencies to 24 , thanks to a campaign involving 6 observatories during $40 \mathrm{~d}$. Again, this can be considered the limit for a multisite campaign which can be organized with a moderate effort. The next step was to set up a campaign not only involving more than 6 sites, bust also spanning a longer time baseline. Moreover, the recent results obtained by Mantegazza \& Poretti (2002) strongly indicated how spec- 


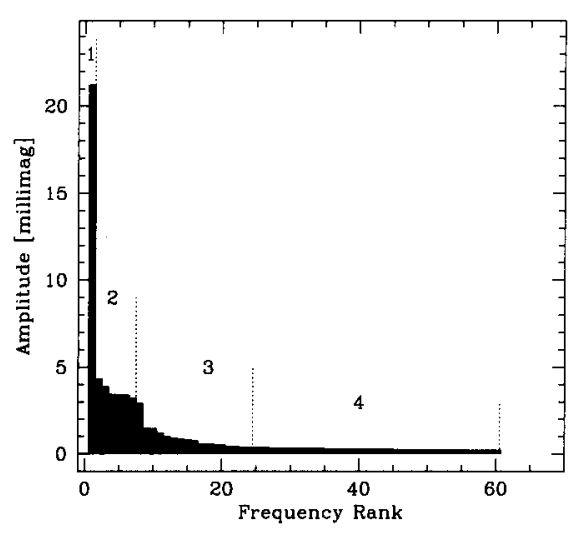

Figure 1. The increasing number of detected frequencies in the FG Vir light curve on the basis of different observational efforts. 1: López de Coca et al. (1984); 2: Mantegazza, Poretti \& Bossi (1994); 3: Breger et al. (1999); 4: Breger (2003, private communication).

troscopy can supply useful hints about the inclination of the rotational axis and to propose some mode identifications. Therefore, the 2002-2003 campaign on FG Vir has been performed by combining spectroscopy and photometry over a 2 -yr time baseline. The aim is to detect close doublets and investigate in a more powerful way the eventual amplitude variations. Such a long-term campaign constitutes the new frontier of the ground-based observations.

Fig. 1 shows how the number of detected frequencies increased by refining the observational approach. From Fig. 1 it can be extrapolated that the lowering of the threshold amplitude by a factor $10^{-1}$ (which should be easily attainable from space) will allow us to detect hundreds of excited modes.

\section{The preparation of the COROT space mission}

The COROT satellite will monitor targets which must be selected in two fields centered at $\alpha=6^{\mathrm{h}} 50^{\mathrm{m}}, \delta=0^{\circ}$ (Anticenter direction), and $\alpha=18^{\mathrm{h}} 50^{\mathrm{m}}, \delta=0^{\circ}$ (Center direction). The prototype of the class, $\delta$ Sct itself, is included in the Center direction field, but it is considered too evolved to match the goals of the mission, as asteroseismic inferences for these stars are greatly complicated by the richness of excited modes in a narrow frequency range ( 275 possible modes in a $4 \mathrm{~d}^{-1}$ interval; Templeton et al. 1997). Therefore, we searched for new $\delta$ Sct stars located in the COROT field-of-view which are unevolved stars (highpriority for COROT) or little evolved ones (low-priority stars). Efforts have been concentrated especially in the galactic Center direction, as there are not many solar-like candidates in such a direction and target allocation is easier. 


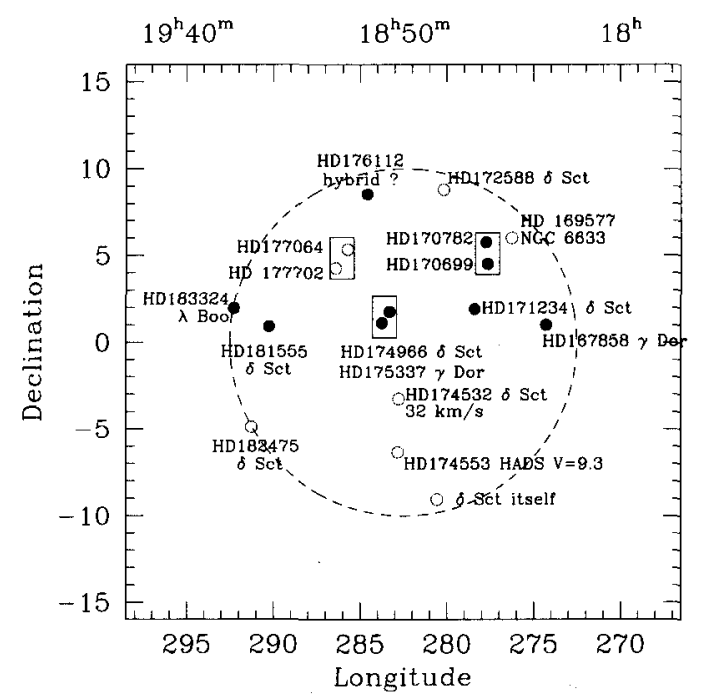

Figure 2. The location of the stars of interest in the field-of-view of COROT, Galactic Center direction. Filled circles are unevolved stars, open circles are evolved ones. Three couples of stars can be observed together in the seismology CCD, shown as small rectangular boxes. The type of variability or some physical characteristic are also reported.

As a result, we actually scanned the solar neighbourhood; $\delta$ Sct stars are commonly found and the incidence of variability is around $23 \%$ (13 variables out of 57 candidates) for stars located in the lower part of the instability strip, i.e., the region between the ZAMS and the blue hooks of the evolutionary tracks (Poretti et al. 2003). With such a percentage, the possibility of finding $\delta$ Sct stars in a limited sky region is reasonable and they can provide a suitable basis of asteroseismic targets for any space mission. Fig. 2 shows the specific result obtained for COROT. The fine tuning of the candidates is now possible and the ground-based preparatory work can greatly take advantage from the expertise gathered in the past decades on the case studies. It is suitable that the $\delta$ Sct stars which will be selected as COROT targets will became case studies for spectroscopy and/or preliminary photometry.

\section{Colour information from space missions}

When performing photometry in different colours it is possible to calculate solutions for each passband. The phase differences and the amplitude ratios will 
be then used to discriminate the different spherical harmonics of a non-radially pulsating star, therefore helping to identify the oscillation mode (Garrido 2000).

The exoplanetary camera of the COROT space mission consists on a CCD with a bi-prism in front of it giving coloured spots on the detector (see G. Vauclair, these proceedings, for details). The three colours basically supplied by the camera are sufficient to distinguish the different spherical degrees $\ell$ of the corresponding radial or non-radial excited periods, making therefore possible the mode identification of several types of pulsating stars, including $\delta$ Scuti stars. Physical information derived from these coloured data also includes limbdarkening coefficients, non-adiabatic phase lags (see Garrido 2003 for details) and, for some specific cases, clues concerning stellar rotation (see DaszynskaDaszkiewicz et al. 2002 for details).

EDDINGTON is an ESA space mission devoted to asteroseismology and to planet detection by the method of transits. The present status of the mission consists of three telescopes: one will receive white light, while the others are equipped with two coloured filters blocking only a small ( 5 to $10 \%$ ) fraction of the light from the red (Ultra-wide Blue Filter) or from the blue (Ultra-wide Red Filter). Differences between the white light and these two coloured filters give narrow enough photometric bands to be useful for mode identification, as shown in detail in Deeg \& Garrido (2003). In this way not many photons are lost, as required by the original mission specifications. Obviously, the system is valid only for stars showing relatively high $\mathrm{S} / \mathrm{N}$ photometric amplitudes (see Garrido 2003 for details). $\delta$ Scuti stars will have sufficient $\mathrm{S} / \mathrm{N}$ ratios to easily derive phases and amplitudes for the different colours.

\section{References}

Breger, M., Handler, G., Garrido, R., et al. 1999, A\&A, 349, 225

Daszynska-Daszkiewicz, J., Dziembowski, W.A., Goupil, M.J. 2002, A\&A, 392, 151.

Deeg, H., Garrido, R., 2003, Eddington Internal Report

Garrido, R. 2000, in Delta Scuti and Related Stars, eds M. Breger \& M.H. Montgomery, ASP Conf. Ser., vol. 210, p. 67

Garrido, R. 2003, 2nd Eddington workshop, Palermo.

López de Coca, P., Garrido, R., Costa, V., Rolland, A. 1984, IBVS 2465

Mantegazza, L., Poretti, E. 2002, A\&A, 396, 911

Mantegazza, L., Poretti, E., Bossi, M. 1994, A\&A, 287, 95

Poretti, E., 2000, in ASP Conf. Ser., Vol. 210, Delta Scuti and Related Stars, eds M. Breger \& M.H. Montgomery (San Francisco: ASP), 45

Poretti, E., Garrido, R., Amado, P.J., et al. 2003, A\&A, 406, 203

Templeton, M., McNamara, B.J., Guzik, J.A. 2003, AJ, 114, 1592 